\title{
Article \\ SOA-Based Multilevel Polarization Shift On-Off Keying Transmission for Free-Space Optical Communication
}

\author{
Yan-Qing Hong $\mathbb{D}^{\mathbb{1}}$, Do-Hoon Kwon, Jae-Young Choi, In-Ho Ha, Won-Ho Shin and Sang-Kook Han * \\ Department of Electrical and Electronics Engineering, Yonsei University, Seoul 03722, Korea; \\ hongy7@yonsei.ac.kr (Y.-Q.H.); ehgns222@yonsei.ac.kr (D.-H.K.); chql@yonsei.ac.kr (J.-Y.C.); \\ 91hainho@yonsei.ac.kr (I.-H.H.); whshin0902@yonsei.ac.kr (W.-H.S.) \\ * Correspondence: skhan@yonsei.ac.kr
}

Citation: Hong, Y.-Q.; Kwon, D.-H.; Choi, J.-Y.; Ha, I.-H.; Shin, W.-H.; Han, S.-K. SOA-Based Multilevel Polarization Shift On-Off Keying Transmission for Free-Space Optical Communication. Photonics 2021, 8 , 100. https://doi.org/10.3390/ photonics 8040100

Received: 19 March 2021

Accepted: 29 March 2021

Published: 31 March 2021

Publisher's Note: MDPI stays neutral with regard to jurisdictional claims in published maps and institutional affiliations.

Copyright: (c) 2021 by the authors. Licensee MDPI, Basel, Switzerland. This article is an open access article distributed under the terms and conditions of the Creative Commons Attribution (CC BY) license (https:// creativecommons.org/licenses/by/ $4.0 /)$.

\begin{abstract}
This paper proposes a semiconductor optical amplifier (SOA)-based multilevel polarization shift on-off keying (MPS-OOK) transmission for free-space optical (FSO) communication. At the transmitter end, the MPS-OOK signal is modulated with a constant linear state of polarization (SOP) at the high-intensity level and various SOPs at the low-intensity level in order to improve the spectral efficiency (SE) with the transmitted power efficiency. At the receiver end, first, a polarizationindependent SOA was introduced to optically suppress the turbulence-induced scintillation effect and equalize the intensities of the various SOPs in the deep gain saturation state without polarization distortion. Then, a linear polarizer (LP) with a high extinction ratio (ER) was deployed to convert the equalized SOPs into a known intensity. Finally, the converted MPS-OOK signal was detected using a single photodiode (PD) and distinguished using a multilevel fixed-threshold decision (M-FTD). The proposed technique was evaluated using experiments. A Mach-Zehnder modulator (MZM)based fading simulator was introduced to emulate the turbulence-induced scintillation effect. The experimental results demonstrated that the scintillation effect was effectively mitigated and the SE was improved by up to $2 \mathrm{bit} / \mathrm{s} / \mathrm{Hz}$ using the proposed four-level polarization shift on-off keying (4PS-OOK) transmission.
\end{abstract}

Keywords: multilevel polarization shift on-off keying; semiconductor optical amplifier; scintillation effect; spectral efficiency; free-space optical communications

\section{Introduction}

Free-space optical (FSO) communication has been widely researched in recent decades due to its characteristics of a high data rate, wide bandwidth, low power consumption, less mass, an unlicensed spectrum, and high security [1]. However, the system performance is impaired by the severe fluctuations in the received signal intensity caused by the atmospheric turbulence-induced beam scintillation effect, which is the major issue in FSO communication [2]. The state of polarization (SOP) maintains a stable state in the atmospheric turbulence channel using a spatially coherent plane wave [3]. Therefore, the application of polarization has been extensively studied to improve the performance of FSO communication systems.

The polarization division multiplexing (PDM) technique has been studied to improve the capacity of FSO communication systems via the application of multiple polarizations $[4,5]$. However, the performance is distorted by the scintillation-effect-induced intensity fluctuations. The polarization shift keying (PolSK) method has been researched to mitigate the scintillation effect via the estimation of SOPs using knowledge of the Stokes parameters [6,7]. However, the performance is significantly degraded in the peak and deep-fading parts of the scintillation effect. Furthermore, PolSK is inefficient in the transmitted optical power compared to on-off keying (OOK). FSO communication is an average-power-limited system due to the average power limitation characteristics of the optical amplifiers [8]. Thus, polarization shift OOK (PS-OOK) was studied in our previous 
work to effectively mitigate the scintillation effect with the transmitted power efficiency [9]. However, the spectral efficiency (SE) of PS-OOK is the same as the conventional OOK due to the application of two SOPs. Higher-order modulation techniques, such as pulseamplitude modulation 4 (PAM-4), multilevel polarization shift keying (MPolSK), and multilevel phase-shift keying (MPSK), have been researched to improve the capacity of FSO communication systems $[5,7,10]$. Therefore, it is preferable to improve the SE of PS-OOK via the transmission of multilevel SOPs.

In this study, we proposed a semiconductor optical amplifier (SOA)-based multilevel polarization shift on-off keying (MPS-OOK) transmission for FSO communication. At the transmitter end, the MPS-OOK signal was modulated with a constant SOP at the highintensity level and various SOPs at the low-intensity level in order to enhance the SE with the transmitted power efficiency. At the receiver end, a polarization-independent SOA and a linear polarizer (LP) were applied to detect the MPS-OOK signal. First, the scintillation effect was optically mitigated using the polarization-independent SOA in the gain saturation state based on nonlinear gain characteristics. Then, the intensities of the various SOPs were equalized in a much deeper gain saturation state without polarization distortion. Then, an LP with a high extinction ratio (ER) was deployed after the SOA to transform the equalized SOPs into the various intensity levels. Finally, the transformed MPS-OOK signal was detected using a single photodiode (PD) and distinguished using a multilevel fixed-threshold decision (M-FTD). The proposed technique was evaluated in experiments under the Mach-Zehnder modulator (MZM)-based fading-simulator-accommodated scintillation effect. The experimental results illustrated that the SE was enhanced by up to $2 \mathrm{bit} / \mathrm{s} / \mathrm{Hz}$ with effective scintillation mitigation using the proposed four-level polarization shift on-off keying (4PS-OOK) transmission.

\section{Operating Principle}

Figure 1 shows the block diagram of the proposed technique. At the transmitter end, the multilevel linear SOPs are modulated into the OOK signal. A laser diode (LD) with a continuous wavelength $(\mathrm{CW})$ is polarized into the linear SOP of $45^{\circ}$ using a polarization controller (PC). The polarized laser beam is split into two orthogonal $x$ - and $y$-polarizations using a polarization beam splitter (PBS). The $s_{-} 1[t]$ and $s_{-} 2[t]$ signals are modulated using MZMs with electrical signals $d \_1[t]$ and $d \_2[t]$, respectively. This is represented by the following:

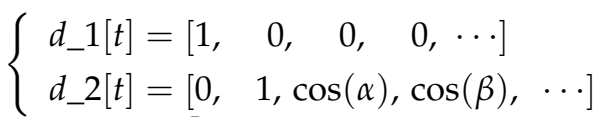

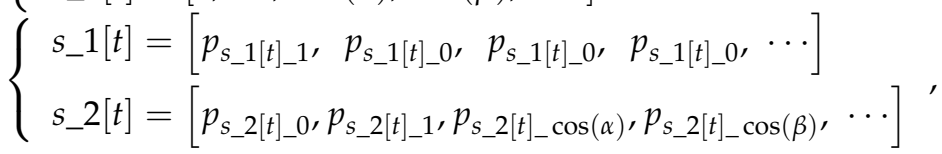

where $p_{s_{-} 1[t]_{-} 1}$ and $p_{s_{-} 1[t]_{-} 0}$ are the bit powers of $s_{-} 1[t]$, and $p_{s_{-} 2[t]_{-} 0}, p_{s_{-}}[t]_{-} 1, p_{s_{-} 2[t]_{-} \cos (\alpha)}$, and $p_{s_{-} 2[t]-\cos (\beta)}$ are the bit powers of $s_{-} 2[t]$. The signal $s_{-} 2[t]$ is attenuated using a variable optical attenuator (VOA). $s_{-} 1[t]$ and the attenuated $s_{-} 2^{\prime}[t]$ are combined using a polarization beam combiner (PBC) to generate the MPS-OOK signal $s[t]$. The SOPs of $s[t]$ were determined by the power ratio between $s_{-} 1[t]$ and $s_{-} 2^{\prime}[t]$, which is given by the following [3]:

$S O P_{s[t]}=\arctan \left(s \_1[t] / s \_2^{\prime}[t]\right) \times 180^{\circ} / \pi=\arctan \left(s \_1[t] /\left(s \_2[t] / G_{V O A}\right)\right) \times 180^{\circ} / \pi$,

where $G_{V O A}$ is the degree of attenuation from the VOA. Therefore, the MPS-OOK signal is modulated with a constant SOP at the high-intensity level and various SOPs at the low-intensity level with the transmitted power efficiency. The ER of the MPS-OOK is determined by $G_{V O A}$, and the SOPs of the MPS-OOK are dependent on $d \_2[t]$, the ER of the MZM, and $G_{V O A}$. The MPS-OOK signal suffers the scintillation effect in the turbulence channel. At the receiver end, the polarization-independent SOA is deployed to mitigate the scintillation effect and equalize the intensities of the various SOPs in the deep gain 
saturation state without the distortion of the SOPs. The scintillation effect is effectively mitigated by the nonlinear gains from the SOA since the high dynamic gain frequency (around $10 \mathrm{GHz}$ ) of the SOA is much higher than the scintillation effect (less than a few $\mathrm{kHz})[1,11]$. Then, the intensities of the various SOPs are equalized by the high dynamic gains in a much deeper gain saturation state. An LP is applied after the SOA to transform the knowledge of the SOPs into various intensity levels. The intensity spacing of the transformed MPS-OOK signal is determined by the degree of the SOPs. The MPS-OOK signal is detected via a single PD and decided using an M-FTD. Consequently, the various SOPs are detected under effective scintillation mitigation using the proposed technique.

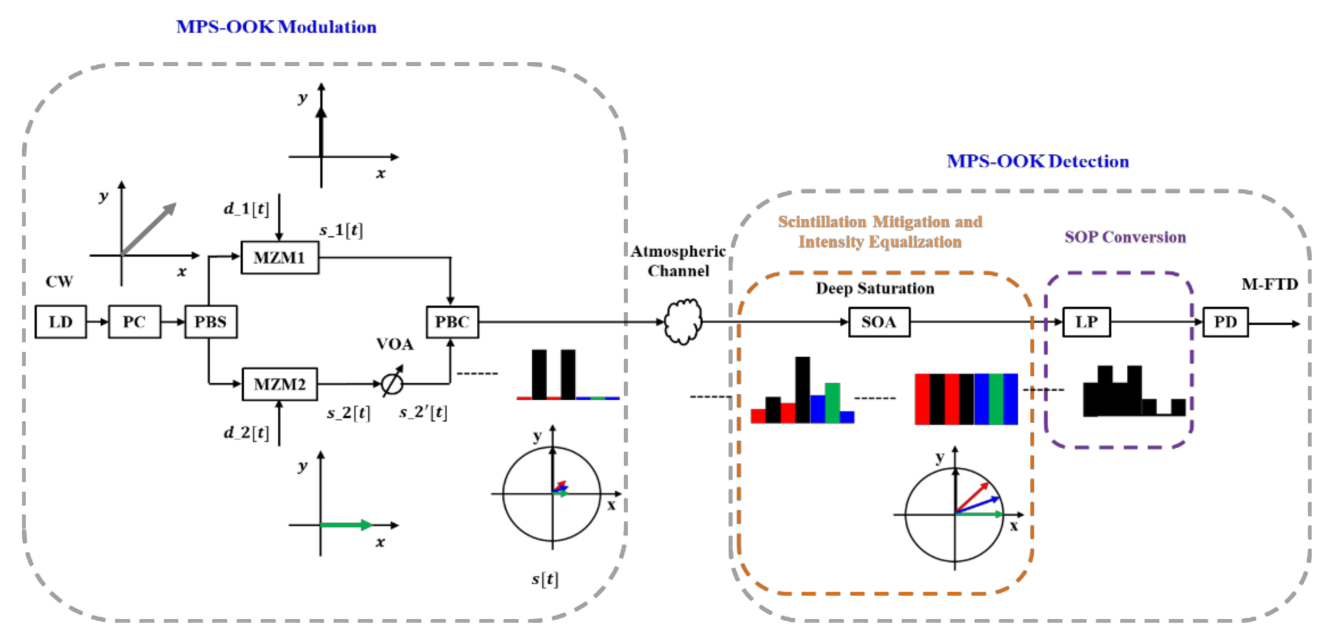

Figure 1. Block diagram of the proposed technique. CW: continuous wavelength, LD: laser diode, LP: linear polarizer, M-FTD: multilevel fixed-threshold decision, MPS-OOK: multilevel polarization shift on-off keying, MZM: Mach-Zehnder modulator, PBC: polarization beam combiner, PBS: polarization beam splitter, PC: polarization controller, PD: photodiode, SOA: semiconductor optical amplifier, SOP: state of polarization, VOA: variable optical attenuator.

\section{Experiments and Results}

The experiments were divided into two parts. The proposed MPS-OOK modulation was verified without the scintillation effect. Furthermore, the proposed SOA-based MPS-OOK detection was compared to the conventional OOK detection under different scintillation effects.

Figure 2a shows the experimental setup of the MPS-OOK modulation. The wavelength of the LD was set to $1550 \mathrm{~nm}$. The SOP was set to $45^{\circ}$ using PC1. A PBS was used to obtain optical beams with orthogonal polarizations. Data1 $d \_1[t]$ and data2 $d \_2[t]$ were imported into the radio-frequency (RF) ports of MZM1 and MZM2, respectively. The bias voltages of MZM1 and MZM2 were set to the quadrature points in order to have large ERs. An optical delay line (ODL) was used to match the bit synchronization between the optical signals from MZM1 and MZM2. VOA1 was set to $15 \mathrm{~dB}$ to have the optimized ER and SOPs for the MPS-OOK signal [9]. An MPS-OOK signal was generated by combining two signal branches using the PBC. Figure $2 \mathrm{~b}$ depicts the experimental setup of the SOP measurement. PC2 was used to match the optical axes between the MPS-OOK and the PBS. VOA2 and VOA3 were applied to measure the average powers of orthogonal polarizations. The PC2 was altered to provide equal values for VOA2 and VOA3 under the transmission of the MPS-OOK with a single SOP of $45^{\circ}$ in order to match the optical axes between the MPSOOK and the PBS. The SOPs of the modulated MPS-OOK were calculated using the power ratio between $\mathrm{VOA} 2$ and $\mathrm{VOA} 3$ via $\mathrm{SOP}=\arctan (\mathrm{VOA} 2 / \mathrm{VOA} 3) \times 180^{\circ} / \pi$. Figure $2 \mathrm{c}$ illustrates the experimental setup of the SOA-based MPS-OOK detection. An MZM-based fading simulator was introduced to emulate the scintillation effect $[9,12]$. The modeled timevarying intensity fluctuation signal and MZM were used to establish the fading simulator. Based on the Kolmogorov power-law spectrum and Taylor frozen turbulence hypotheses, 
the time-varying intensity fluctuations were generated from the temporal spectrum of the log-amplitude fluctuations [13]. Then, the random phase information was modulated into the temporal spectrum. Then, it was transformed into the time domain using an inverse Fourier transform. Finally, the normalized time-varying intensity fluctuations signal was modeled using first-order Rytov approximations $[9,14]$. The modeled signal was imported into the RF port of the MZM to accommodate the scintillation effect into the traveling optical signal. The degree of the scintillation effect was measured in terms of the scintillation index $\sigma_{I}^{2}=\left\langle I^{2}\right\rangle /\langle I\rangle^{2}-1$, where $\sigma_{I}^{2}$ indicates the normalized variance of the intensity fluctuations [14]. Figure 3a illustrates the MZM-based fading-simulator-emulated scintillation effect with a $\sigma_{I}^{2}$ of 0.11 in the time domain. A time-varying intensity fluctuation signal was observed. Figure $3 b$ illustrates the frequency domain of the accommodated scintillation effect, which was dominated by the low-frequency components. Figure $3 c$ shows that the accommodated scintillation effect fit well with the lognormal distribution, which is the widely used statistical model for weak turbulence. Therefore, the scintillation effect was effectively emulated by the MZM-based fading simulator. Since SOPs keep a stable state in the turbulence channel, the MPS-OOK signal suffers the scintillation-effectinduced intensity fluctuations. Thus, it was sufficient to emulate the turbulence channel using the MZM-based fading simulator in this study.

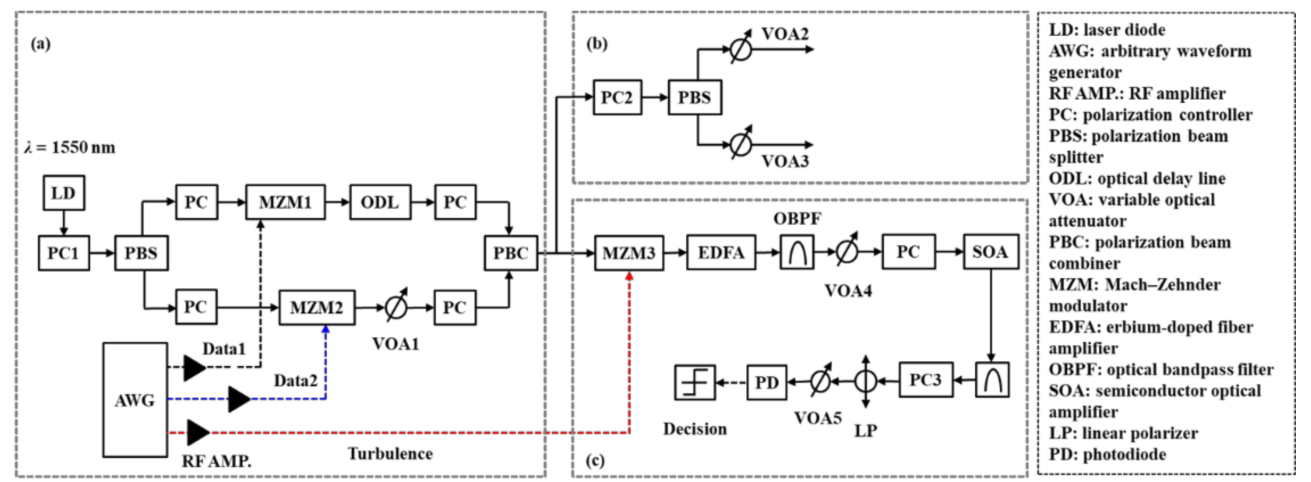

Figure 2. Experimental setup of the (a) MPS-OOK modulation, (b) SOP measurement, and (c) SOA-based MPS-OOK detection.
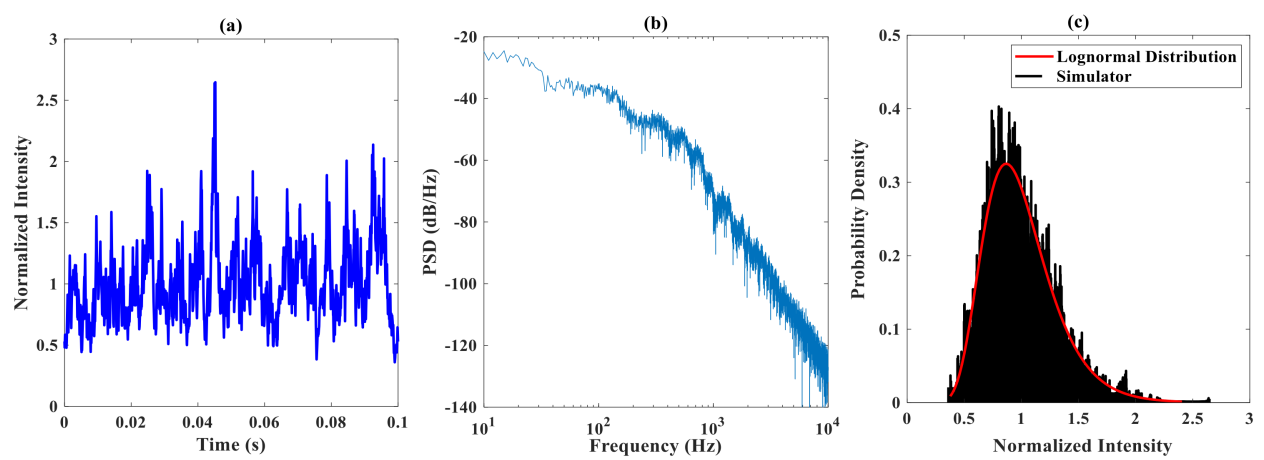

Figure 3. MZM-based fading-simulator-emulated scintillation effect with a $\sigma_{I}^{2}$ of 0.11 : (a) time domain, (b) frequency domain, and (c) distribution.

An erbium-doped fiber amplifier (EDFA) was applied to improve the received optical power. The polarization-independent SOA was applied to optically mitigate the scintillation effect and equalize the intensities of the various SOPs in the deep gain saturation region. PC3 was deployed to match the optical axis between the MPS-OOK and the LP (Fiber-Optic In-Line Polarizer, POL-001, General Photonics, CA, USA). The LP was used to convert the various SOPs into different intensity levels. The transformed MPS-OOK signal was detected using a single PD and decided using an M-FTD. The symbol rate was set to $1.25 \mathrm{Gsymbol} / \mathrm{s}$, as shown in Table 1. 
Table 1. Parameters used in the experiments.

\begin{tabular}{cc}
\hline Parameters & Values \\
\hline Wavelength & $1550 \mathrm{~nm}$ \\
\hline Bias voltage of the MZM & Quadrature point \\
\hline Turbulence channel & MZM-based fading simulator \\
\hline$\sigma_{I}^{2}$ & $0.11,0.59$ \\
\hline VOA1 & $15 \mathrm{~dB}$ \\
\hline Symbol rate & $1.25 \mathrm{Gsymbol} / \mathrm{s}$ \\
\hline
\end{tabular}

Tables 2 and 3 illustrate the measurement of SOPs of the modulated three-level polarization shift on-off keying (3PS-OOK) and 4PS-OOK. $d \_1[t]$ and $d \_2[t]$ were coded according to Equations (1) and (2). The SOPs of the modulated 3PS-OOK and 4PS-OOK were calculated using the power ratio between VOA2 and VOA3. Regarding 3PS-OOK, the high-intensity level was modulated with SOPs of $0^{\circ}$ and the low-intensity level was modulated with SOPs of $60^{\circ}$ and $85^{\circ}$. Regarding 4PS-OOK, the high-intensity level was modulated with SOPs of $0^{\circ}$ and the low-intensity level was modulated with SOPs of $48.2^{\circ}$, $70.5^{\circ}$, and $85^{\circ}$.

Table 2. SOPs of three-level polarization shift on-off keying (3PS-OOK).

\begin{tabular}{cccc}
\hline$d \_1[t]$ & 1 & 0 & 0 \\
\hline$d \_2[t]$ & 0 & 0.15 & 1 \\
\hline SOP & $0^{\circ}$ & $60^{\circ}$ & $85^{\circ}$ \\
\hline
\end{tabular}

Table 3. SOPs of the four-level polarization shift on-off keying (4PS-OOK).

\begin{tabular}{ccccc}
\hline$d \_1[t]$ & 1 & 0 & 0 & 0 \\
\hline$d \_2[t]$ & 0 & 0.1 & 0.25 & 1 \\
\hline SOP & $0^{\circ}$ & $48.2^{\circ}$ & $70.5^{\circ}$ & $85^{\circ}$ \\
\hline
\end{tabular}

Figure 4 shows the eye diagrams of the proposed 3PS-OOK and 4PS-OOK transmissions. The experiments were conducted without the scintillation effect to provide a clear measurement of the eye patterns. The average input power at the PD was set to $-3 \mathrm{dBm}$ using VOA5 to reduce the PD noise. The eye patterns of the OOK were observed for the modulated 3PS-OOK and 4PS-OOK. The average input power at the SOA was configured to $2 \mathrm{dBm}$ using VOA4 in order to have a deep gain saturation. The eye diagrams after the SOA show that the intensities of the various SOPs were effectively equalized by the gain-saturated SOA. The eye diagrams of the 3PS-OOK and 4PS-OOK were measured after the LP. Two and three levels of nearly balanced vertical eye-opening were observed for the 3PS-OOK and 4PS-OOK, respectively. Thus, the knowledge of the SOPs was effectively transformed into the various intensity levels. Therefore, the 3PS-OOK and 4PS-OOK were effectively modulated and detected using the proposed technique. 


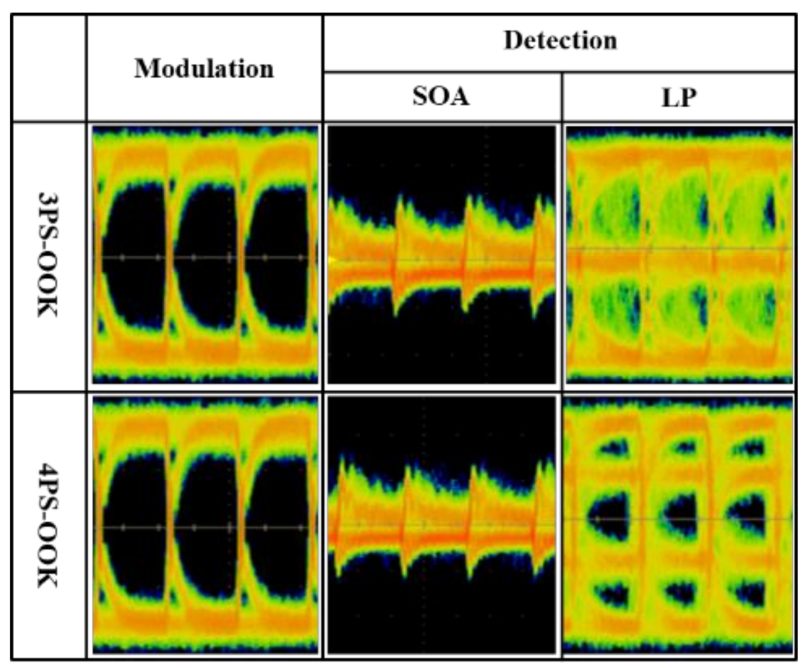

Figure 4. Eye diagrams of the proposed 3PS-OOK and 4PS-OOK transmissions without the scintillation effect.

Figure 5 shows the bit error rate (BER) performance of the OOK, two-level polarization shift on-off keying (2PS-OOK), 3PS-OOK, and 4PS-OOK transmissions under $\sigma_{I}^{2}$ 's of 0.11 and 0.59 . The scintillation effect was accommodated using the MZM-based fading simulator. The BERs were calculated using a fixed-threshold decision (FTD) under the variation of the average input powers at the SOA. The average input power at the PD was set to $-3 \mathrm{dBm}$ using VOA5 to reduce the PD noise. Regarding the OOK transmission, the BERs were initially enhanced in the gain saturation state of the SOA due to the scintillation effect mitigation by the nonlinear gains. However, the BERs were decreased in the deeper gain saturation state due to the ER degradation of the OOK during the process of scintillation mitigation. Regarding the 2PS-OOK transmission, the BERs were improved with the increase of the average input powers at the SOA since the degraded ER was recovered by the filtering of the polarization of bit " 0 " using the LP. Regarding the 3PS-OOK and 4PS-OOK transmissions, the intensities of the various SOPs were equalized by the SOA in the deep gain saturation state, and the equalized SOPs were converted into the different intensity levels by the LP. Thus, the BERs of the 3PS-OOK and 4PS-OOK were improved with the increase of the average input powers at the SOA. Poor BER performances of $3 \mathrm{PS}-\mathrm{OOK}$ and 4PS-OOK were observed for lower average input powers at the SOA due to the ineffective intensity equalization of the various SOPs. The BERs of the 3PS-OOK and $4 \mathrm{PS}-\mathrm{OOK}$ were close to the 2PS-OOK for the higher average input powers at the SOA due to the effective intensity equalization of the various SOPs in the deep gain saturation state. Furthermore, much higher average input powers at the SOA were required for a $\sigma_{I}^{2}$ of 0.59 compared to a $\sigma_{I}^{2}$ of 0.11 since a deeper gain saturation was needed for a $\sigma_{I}^{2}$ of 0.59 in order to effectively mitigate the scintillation effect. Figure 6 shows the SEs of the proposed technique at a BER of $1 \times 10^{-3}$. The received MPS-OOK signal had a high signal-to-noise ratio (SNR) due to the large output power at the SOA; therefore, the SE of the proposed technique was dependent on the average input powers at the SOA and the strength of the scintillation effect. The SE was calculated using $\log _{2}^{M}$ under the effective scintillation mitigation, where $M$ indicates the number of levels of the SOP $[15,16]$. For the average input power at the SOA of less than $-4 \mathrm{dBm}$, the SE was similar to the OOK and 2PS-OOK with $\sigma_{I}^{2 \prime}$ s of 0.11 and 0.59 . Regarding the average input power at the SOA of $-2 \mathrm{dBm}$, the SE was improved by up to $2 \mathrm{bit} / \mathrm{s} / \mathrm{Hz}$ with a $\sigma_{I}^{2}$ of 0.11 . For an average input power at the SOA of $6 \mathrm{dBm}$, the SE was enhanced by up to $2 \mathrm{bit} / \mathrm{s} / \mathrm{Hz}$ with an $\sigma_{I}^{2}$ of 0.59 as well. Consequently, the SE was improved by up to $2 \mathrm{bit} / \mathrm{s} / \mathrm{Hz}$ under effective scintillation mitigation using the proposed technique with the average input power at the SOA of $6 \mathrm{dBm}$ and a symbol rate of $1.25 \mathrm{Gsymbol} / \mathrm{s}$. The SE can be improved by coherent detection instead of direct detection using an M-FTD, and it can be further enhanced by 
using an MZM with a larger ER, an SOA with lower amplified spontaneous emission (ASE) noises, an LD with a narrower linewidth, and a PD with a higher sensitivity $[15,17]$.
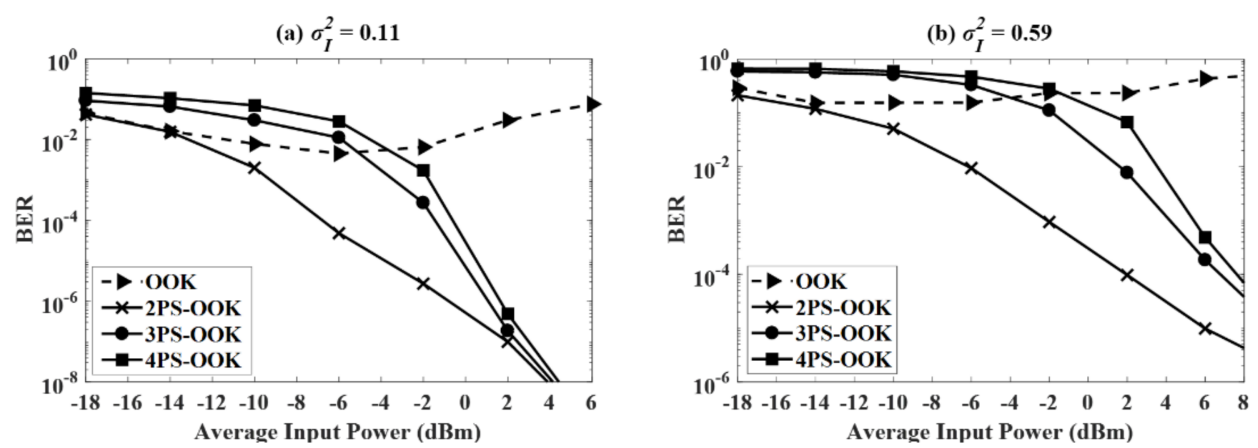

Figure 5. Bit error rate (BER) performance of the on-off keying (OOK), two-level polarization shift on-off keying (2PS-OOK), 3PS-OOK, and 4PS-OOK transmission: (a) $\sigma_{I}^{2}$ of 0.11 and (b) $\sigma_{I}^{2}$ of 0.59 .

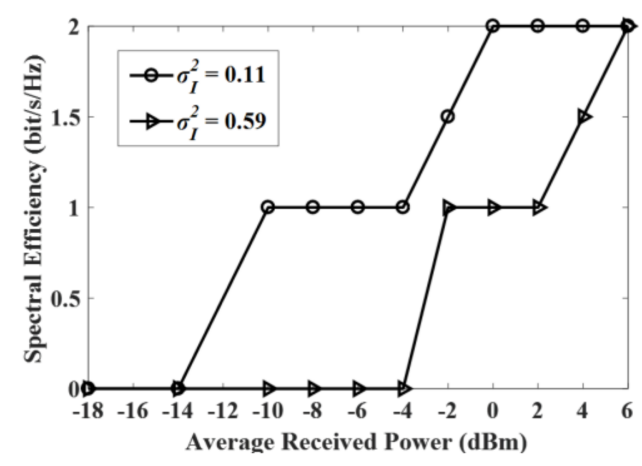

Figure 6. Spectral efficiencies (SEs) of the proposed technique at a BER of $1 \times 10^{-3}$.

\section{Conclusions}

In summary, we proposed an SOA-based MPS-OOK transmission for FSO communication. At the transmitter end, an MPS-OOK signal was modulated with a constant SOP at the high-intensity level and various SOPs at the low-intensity level for the sake of the SE enhancement with the transmitted power efficiency. At the receiver end, a polarization-independent SOA was used to optically suppress the scintillation effect and equalize the intensities of the various SOPs in the deep gain saturation state using the high dynamic nonlinear gains without the polarization distortion. An LP with a high ER was applied after the SOA to convert the equalized SOPs into the different intensity levels. The converted MPS-OOK signal was detected using a single PD and distinguished using an M-FTD. The proposed technique was verified via experiments. An MZM-based fading simulator was used to accommodate the different scintillation effects. The experimental results demonstrated that the SE was enhanced up to $2 \mathrm{bit} / \mathrm{s} / \mathrm{Hz}$ with effective scintillation mitigation using the proposed technique. Therefore, it has high potential and is a feasible technique for FSO communication.

Author Contributions: Conceptualization, Y.-Q.H.; data curation, Y.-Q.H.; formal analysis, Y.-Q.H., D.-H.K., J.-Y.C., I.-H.H. and W.-H.S.; project administration, S.-K.H.; writing-original draft, Y.-Q.H.; writing - review and editing, Y.-Q.H. All authors have read and agreed to the published version of the manuscript.

Funding: This work was supported by the Institute for Information and Communications Technology Promotion (IITP) grant funded by the Korean government (MSIT; Ministry of Science and ICT) (No. 2019-0-00685, Free-space-optical-communication-based vertical mobile network).

Institutional Review Board Statement: Not applicable. 
Informed Consent Statement: Not applicable.

Data Availability Statement: Not applicable.

Conflicts of Interest: The authors declare no conflict of interest.

\section{References}

1. Kaushal, H.; Kaddoum, G. Optical communication in space: Challenges and mitigation techniques. IEEE Commun. Surv. Tutor. 2017, 19, 57-96. [CrossRef]

2. Zhu, X.; Kahn, J.M. Free-space optical communication through atmospheric turbulence channels. IEEE Trans. Commun. 2002, 50, 1293-1300.

3. James, D.F.V. Change of polarization of light beams on propagation in free space. J. Opt. Soc. Am. A 1994, 11, 1641-1643. [CrossRef]

4. Tsai, W.S.; Lu, H.H.; Huang, Y.C.; Tu, S.C.; Huang, Q.P. A PDM-based bi-directional fibre-FSO integration with two RSOAs scheme. Sci. Rep. 2019, 9, 8317. [CrossRef] [PubMed]

5. Zhang, R.; Peng, P.C.; Li, X.; Liu, S.; Zhou, Q.; He, J.; Chen, Y.W.; Shen, S.; Yao, S.; Chang, G.K. 4×100-Gb/s PAM-4 FSO transmission based on polarization modulation and direct detection. IEEE Photon. Technol. Lett. 2019, 31, 755-758. [CrossRef]

6. Benedetto, S.; Gaudino, R. Direct detection of optical digital transmission based on polarization shift keying modulation. IEEE J. Sel. Areas Commun. 1995, 13, 531-542. [CrossRef]

7. Zhang, X.; Ghassemlooy, Z.; Rajbhandari, S.; Popoola, W.O.; Lee, C.G. Coherent heterodyne multilevel polarization shift keying with spatial diversity in a free-space optical turbulence channel. J. Lightw. Technol. 2012, 30, 2689-2695.

8. Caplan, D.O.; Carney, J.J.; Fitzgerald, J.J.; Gaschits, I.; Kaminsky, R.; Lund, G.; Hamilton, S.A.; Magliocco, R.J.; Murphy, R.J.; Rao, H.G.; et al. Multi-rate DPSK optical transceivers for free-space applications. In Free-Space Laser Communication and Atmospheric Propagation XXX; SPIE: Bellingham, WA, USA, 2014; Volume 8971.

9. Hong, Y.Q.; Shin, W.H.; Han, S.K. Enhancement of SOA-based scintillation mitigation by PS-OOK transmission in FSO communication. IEEE Photonics J. 2020, 12, 7903510. [CrossRef]

10. Chen, C.; Zhong, W.D.; Li, X.; Wu, D. MDPSK-Based Nonequalization OFDM for coherent free-space optical communication. IEEE Photon. Technol. Lett. 2014, 26, 1617-1620. [CrossRef]

11. Yiannopoulos, K.; Sagias, N.C.; Boucouvalas, A.C. On the performance of semiconductor optical amplifier-assisted outdoor optical wireless links. IEEE J. Sel. Areas Commun. 2015, 33, 1869-1876.

12. Takashi, S.; Morio, T.; Hideki, T. Fading simulator for Satellite-to-ground optical communication. J. Natl. Inst. Inf. Commun. Technol. 2012, 59, 95-102.

13. Shen, H.; Yu, L.; Fan, C. Temporal spectrum of atmospheric scintillation and the effects of aperture averaging and time averaging. Opt. Commun. 2014, 330, 160-164. [CrossRef]

14. Toyoshima, M.; Takenaka, H.; Takayama, Y. Atmospheric turbulence-induced fading channel model for space-to-ground laser communications links. Opt. Express 2011, 19, 15966-15975. [CrossRef] [PubMed]

15. Al-Jarrah, M.A.; Al-Dweik, A.; Park, K.H.; Alouini, M.S. Amplitude-coherent detection for optical wireless communications: Opportunities and limitations. IEEE Open J. Commun. Soc. 2020, 1, 550-562. [CrossRef]

16. Chaaban, A.; Hranilovic, S. Capacity of optical wireless communication channels. Phil. Trans. R. Soc. A 2019, 378, 2169. [CrossRef] [PubMed]

17. Hu, W.X.; Wei, Z.; Popov, S.; Leeson, M.; Zhang, M.; Xu, T. Non-coherent detection for ultraviolet communications with inter-symbol interference. J. Lightw. Technol. 2020, 38, 4699-4707. [CrossRef] 